\title{
Preservation of Nuclear Envelope and Pore Complex Dynamics using High Pressure Freezing and Cryo-Field Emission SEM.
}

\author{
T.D. Allen, M.Hughes, S.A. Rutherford, S. Murray, S.Bagley, E. Kiseleva, M.W. Goldberg ${ }^{1}$ \\ Structural Cell Biology, Paterson Institute for Cancer Research, \\ Christie Hospital, Manchester M20 9BX, UK. \\ ${ }^{1}$ Science Laboratories, University of Durham, South Road, Durham, \\ County Durham DH1 3LE, UK.
}

Studies over the last decade have led to a significant increase in our understanding of many of the structural elements of the nuclear pore complex (NPC) and the soluble factors involved in the controlled passage of import and export complexes [1]. Currently however, the direct interactions between complexes 'in transit' and the fixed structural elements of the NPC remain uncharacterised. There is also controversy over the diameter of the channel through the centre of the NPC. Most structural NPC studies, whether TEM, cryo-TEM or FESEM, indicate a central structure in the pore termed a transporter, through which a central channel of around $26 \mathrm{nms}$ maximum diameter has been established by microinjection of colloidal gold particles coated with nucleophilic proteins. Recent findings suggest that virus particles of around 40nms in diameter can pass directly through the pore. This raises the possibility that there may be a potential for expansion of the central channel through the transporter. We have pursued most of our previous studies employing conventional chemical preservation for both TEM and FESEM visualisation of NPC structure, but the standard structural elements, including the transporter, are common to both chemical preservation and 'thin ice' unfixed 3D reconstructions from the TEM. However, most of the structural information on vertebrate NPCs emanates from isolated nuclear envelopes from the giant nuclei of amphibian oocytes which have been produced by nuclear isolation and nuclear envelope separation, prior to whatever preservation protocol was used subsequently. Although reports do exist that active translocation through the NPCs in these isolated envelopes can be retained in the right experimental conditions [2], an approach of rapid stabilisation 'in situ' of the nuclear envelope without isolation may well produce direct structural evidence relevant to the physiological transport state of the nuclear pore.

Our own current approach is based on rapid freezing, followed by surface imaging of cryohydrated nuclear envelopes and nuclear pores. Initially we are using isolated nuclear envelopes mounted on carbon-formvar support films and plunge frozen in liquid ethane, or on $3 \mathrm{~mm}$ sapphire discs frozen in a high pressure freezer (Baltec HPM 010). Nuclear envelopes are either frozen directly in isolation buffer, or briefly rinsed in distilled $\mathrm{H}_{2} \mathrm{O}$ directly before freezing. After transferring the frozen grids or sapphire discs to a Gatan CT 3500 cryo-rod, the rod itself is inserted into a dedicated holder for etching and chromium coating on an Edwards Auto 306 coating unit with cryopump [3]. The cryorod itself incorporates an environmental chamber into which the specimen can be withdrawn to prevent frosting during transfer between the specimen mounting jig and the coating unit, and subsequently the coating unit and the airlock for the 'in lens' specimen position in the SEM. Prior to coating, the specimens are surrounded in a liquid $\mathrm{N}_{2}$ cooled tubular anticontaminator within the coating unit. Current experiments indicate that an etching time of around 10-15 minutes at a vacuum of $2 \times 10^{-7}$ mbar and a specimen temperature of 
$-100^{\circ} \mathrm{C}$ results in some areas of the isolated nuclear envelopes which retain a cryo-hydrated state rather than becoming freeze dried [4]. At this point the specimens are sputter coated with 4nms of chromium, prior to venting the work chamber of the coating unit to dry nitrogen, and withdrawing the specimen into the evacuated environmental chamber of the cryo-rod for transfer to the top stage of a Topcon DS130F 'in lens' field emission SEM.

Although this protocol allows imaging of unfixed isolated nuclear envelopes by in lens FESEM, we are also attempting to directly image the nuclear surface of whole rapidly frozen oocytes. This approach should allow a direct 'in situ' visualisation of unfixed, cryo hydrated nuclear pore complexes which have been stabilised in a few milliseconds by the high pressure freezing of an isolated oocyte. Use of smaller (stage III) oocytes should allow the $200 \mu \mathrm{ms}$ of rapid vitrefication to approach and include the nuclear envelope, as we have demonstrated by freeze substitution and thin sectioning in TEM. Cryo-fracturing of these preparations, however, does not produce surface exposure of the NPCs as the fracture plane closely follows the surface of the nuclear envelope membranes and 'loses' the surface visualisation of the pore complex. In an effort to overcome this, we are exploring 'cryo-planing' and 'cryo-abrasion' of the frozen oocyte using the cryo ultrotome or lapping paper to remove cytoplasm approximately down to the surface of the nucleus, and then etching this surface prior to cryo-coating and examination in the cryohydrated state in the FESEM. Initial experiments with fixed material, frozen and cryo-planed or cryo-abraded prior to critical point drying and room temperature examination, have shown that both the surface of endoplasmic reticulum and nuclear envelope, as well as internal nuclear elements, such as chromatin and nucleo-skeletal elements can be visualised at high resolution in this way, both in isolated oocytes,(Fig 1) and tissue culture monolayers (Fig 2).

\section{References}

[1] T.D. Allen et al., J. Cell Sci. (2000) 113..

[2] R. Peters et al., J. Struct. Biol. (2002) 268.

[3] T.D. Allen et al., Microsc. Microanal. 7 (2001) 724.

[4] R. Apkarian, Microsc. Microanal. 5 (1999) 197
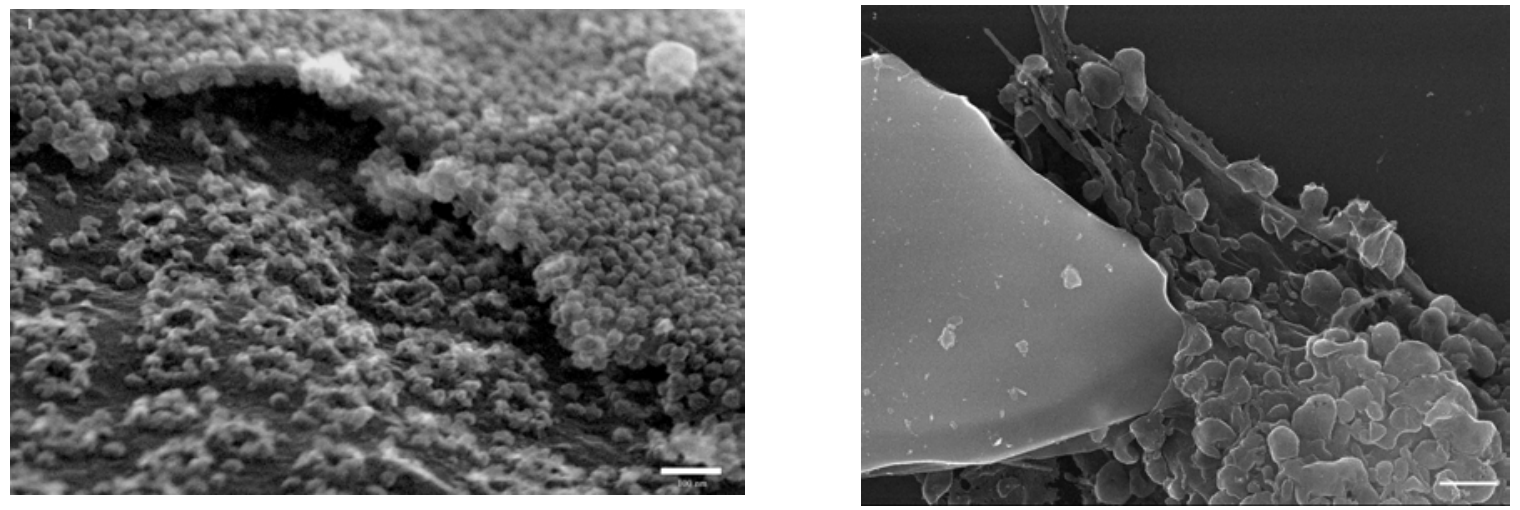

Fig 1 Exposure of nuclear surface of Xenopus oocyte by cryoplaning, showing NPCs and overlaying endoplasmic reticulum with densely packed ribosomes. Scale bar 100nms

Fig 2 Interaction of fragment of abrasive with cell surface during cryo-abrasion. The edge of the abrasive particle has remained embedded in the cell membrane (centre)Scale bar $1.25 \mathrm{mns}$ 